\title{
OBSERVATIONS OF HF PROPAGATION ON A PATH ALIGNED ALONG THE MID-LATITUDE TROUGH
}

\author{
A.J. Stocker, N.Y. Zaalov, E.M. Warrington, and D.R. Siddle \\ Department of Engineering, University of Leicester, Leicester, LE1 7RH, UK \\ emw@le.ac.uk
}

\begin{abstract}
Observations of the direction of arrival and time of flight of HF signals propagating on a $1400 \mathrm{~km}$ path oriented along the mid-latitude trough are presented. At night, the signal commonly arrives from directions offset from the great circle bearing by up to $80^{\circ}$ and these events have been categorised into five main types. Statistics indicating how often these categories of propagation were observed in the period August 2006 to September 2007 are presented. The physical mechanisms which result in the off great circle propagation are also discussed.
\end{abstract}

\section{$1 \quad$ Introduction}

The mid-latitude trough is a region of depleted electron density in the nighttime Fregion ionosphere in which the critical frequencies drop by a factor of at least two and the altitude of the electron density peak rises by $100 \mathrm{~km}$ or more (Moffett and Quegan, 1983). During the winter and equinoctial months, the trough takes the form of a band a few degrees wide in latitude to the equatorward side of the auroral oval, stretching in local time from dusk to dawn. In summer, the trough is much less pronounced and is confined to the hours around midnight. The location of the trough also depends on geomagnetic activity with the region of electron density depletion moving equatorwards and the evening sector tending to move to earlier local times as activity increases.

For terrestrial HF radio systems, the electron density depletion in the trough region reduces the maximum frequency that can be reflected by the ionosphere along the great circle path (GCP). For long paths, the signal is often received via a ground / sea-scatter mechanism to the side of the GCP (Stocker et al., 2003). For shorter paths, gradients in electron density associated with the trough walls and embedded ionospheric irregularities often result in propagation in which the signal path is well displaced from the great circle direction, with directions of arrival at the receiver offset by up to $100^{\circ}$ (Rogers et $a l ., 1997)$. Deviations from the great circle

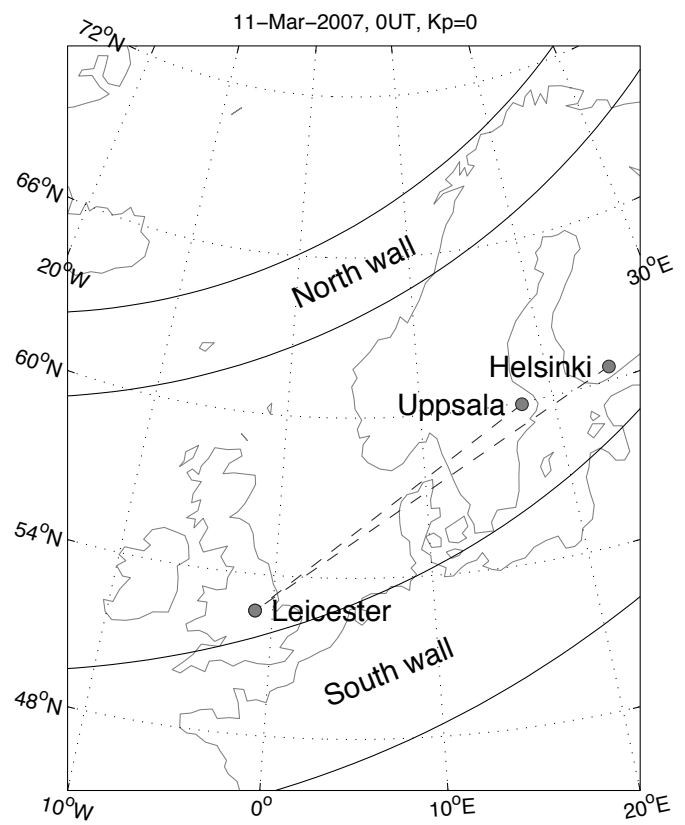

Figure 1. A map showing the paths between Uppsala and Leicester and Helsinki and Leicester together with a typical position for the trough, as estimated by the model of Halcrow and Nisbet (1977) for 0000 UT on 11 March 2006. The four lines indicate the outer and inner edges of the north and south walls. 
direction impact not only on radiolocation systems for which estimates of a transmitter location are obtained by triangulation from a number of receiving sites, but also on any radio communications system in which directional antennas are employed. Furthermore, the Doppler and multi-mode delay spread characteristics of the signal are also affected when propagation is via scatter / reflection from irregularities in or close to the north wall of the trough (Warrington et al., 1997; Warrington and Stocker, 2003).

\section{Observations}

Recently, measurements have been made of the direction of arrival (DOA) and the time of flight (TOF) of HF signals received over two paths oriented along the trough between Uppsala, Sweden (since August 2006) and Helsinki, Finland (since December 2006) and the University of Leicester's field site near to Leicester (see Figure 1). In this paper, only observations from the Uppsala-Leicester path will be presented since these can be compared to similar measurements taken on this path close to solar maximum (the current interval is close to the solar minimum) and for reasons of brevity. Six frequencies were employed, namely 4.6, 7.0, 8.0, 10.4, 11.1, and 14.4 MHz. Each transmission comprised a 13-bit Barker-coded BPSK sequence synchronised to GPS so that the direction of arrival and signal strength could be measured as a function of absolute time of flight. A measurement for a given transmitter and frequency was taken every two minutes. Examination of the 14 months of observations currently available has enabled five types of off great circle propagation events to be identified. Each type is described below with example observations presented in Figures 2 to 6 . The signal reflection points for the same observations given in Figures 2 to 6 are presented in Figure 7. The reflection points have been derived using the TOF and DOA measurements assuming propagation via a single, mirror-like reflection and allowing the reflection height to vary freely. The raw data has been smoothed by the application of a 20-minute running median in each of the three parameters (TOF, azimuth, and elevation).

Type 1: These are characterised at onset by a large sudden increase in time of flight accompanied by a sudden deviation of the bearing to the north. Subsequently, the time of flight decreases over a period of hours while the azimuth either remains fairly constant or returns slowly to close to the great circle direction. In the example presented in Figure 2, there is a second occurrence around 03:00 UT and what may be a second hop mode at 04:30 UT. Type 1 events were commonly observed, except in the summer, in an earlier (2001 - close to solar maximum) experiment (see Siddle et al., 2004a for a number of examples) when they also tended to be longer-lived and were observed on a wider range of frequencies. Theoretical considerations, and the reflection points presented in Figure 7, indicate that these events are consistent with the signal scattering from irregularities in the poleward wall of the trough or from the auroral region (Siddle et al., 2004b).

Type 2: The time of flight gradually increases and is accompanied by a gradual deviation of the azimuth to the north (e.g. see Figure 3). At the same time, there tends to be a decrease in the elevation (although this doesn't occur in all cases). The rate of change of time of flight and azimuth tends to be fairly constant until, after a period of up to a few hours, the signal is no longer detected. At the beginning of the event, instead of a gradual change there can be a sudden, small, increase in time of flight and azimuth deviation. This behaviour is then distinguished from a Type 1 event by the subsequent increase of the azimuth deviation and time of flight. The reflection point (see Figure 7) 
starts clustered around the path mid-point and gradually moves to the north, the signal being lost when it nears the poleward wall of the trough.

Type 3: The time of flight gradually increases and is accompanied by either a gradual southward (Type 3S, e.g. see Figure 4; although note that in this particular case a signal arriving from the north is also sometimes observed) or northward (Type $3 \mathrm{~N}$ ) deviation of the azimuth. After a few hours either the signal is no longer detected (as in Figure 4) or the azimuth slowly returns to the great circle direction (this distinguishes Type $3 \mathrm{~N}$ propagation from Type 2). Although the elevation angle gradually increases in the interval prior to the azimuth going off circle at about 23:30 UT, after that time the elevation angle remains fairly constant. At the start of the interval, the reflection point (Figure 7) is centred on the path midpoint, but once the signal goes off great circle it reflects from a region close to the equatorward wall of the trough.

Type 4: This type of propagation is characterised by a sudden large increase in the time of flight and a strong deviation to the south (typically the azimuth is around $120^{\circ}$ ). Although the signal is usually relatively weak, the example in Figure 5 is a comparatively strong case and is observed at the same time as on-GC propagation. Although the reflection points (Figure 7) are well to the south, the height of reflection given by the simple model used is much too high to be realistic. This, together with the fact that the elevation is similar for both the on and off-GC modes, is consistent with ground sidescatter with a ground reflection a few hundred kilometres to the north of that given in Figure 7 (i.e. in the vicinity of the Alps).

Type 5: This event is similar to the Type 1 event, except that although there is a sudden increase in the time of flight, the signal is deviated to the south instead of the north (see Figure 6). Over the next few hours the azimuth usually returns to the great circle direction (as in Figure 6). In this example, the elevation of the on-great circle signal has been poorly determined (these are the points at zero degrees) probably as a result of the relatively low elevation angle of this E-region mode. However, the elevation for the offgreat circle propagation appears to decrease as the azimuth deviation decreases (particularly in the interval from 23:00-01:00 UT). In Figure 7, the signal is reflected from a region close the equatorward wall of the trough.

The occurrence (as a percentage of nights of observation) of Types 1, 2, and 4 as a function of season and frequency is presented in Figure 8. The seasons have been defined according to ITU recommendation P1148 (1997), with spring including all of March and April, summer including May to August, autumn September and October and winter November to February. The results for summer include data from August 2006 and May-August 2007, while those for autumn include data from SeptemberOctober 2006 and September 2007. In Figure 8, Type 1 events are most common in spring with no clear trend with frequency (except that they are rarely observed at $14.36 \mathrm{MHz}$ ). For $4.64 \mathrm{MHz}$, this type of propagation is also common in winter, while for most other frequencies it occurs least often in winter. This behaviour may be contrasted with that observed in 2001 (see Siddle et al, 2004a;b) where Type 1 propagation was frequently observed ( $40-70 \%$ of nights depending on frequency) except in summer. Furthermore, the events observed in 2001 tended to consist of larger changes in time of flight and azimuth and to be of longer duration. Type 2 events tend to occur more frequently in spring and, for frequencies in the range $8.01-11.12 \mathrm{MHz}$, in the summer. However, for $6.95 \mathrm{MHz}$, there is little other seasonal variation, while at 4.64 and $14.36 \mathrm{MHz}$ the events are rarely observed. In spring, the occurrence strongly 
depends on frequency, peaking at $10.39 \mathrm{MHz}$. Type 4 events are also a springtime phenomenon, being observed on over $30 \%$ of nights for 10.39 and $11.12 \mathrm{MHz}$, but rarely at other frequencies. Of the propagation events not included in Figure 8, Type 3 events are generally rare $(<2 \%)$ for all frequencies except at $4.64 \mathrm{MHz}$ where they are observed $\sim 7 \%$ of nights during the summer. Type 5 propagation is also found on low frequencies, with it being observed on $20 \%$ of winter nights and $8 \%$ of nights in the spring at $4.64 \mathrm{MHz}$.

The occurrence as a function of frequency and $\mathrm{K}_{\mathrm{p}}$ for propagation Types 1, 2, and 4 is presented in Figure 9. Since the off-great circle events tend to occur before midnight (UT), the $K_{p}$ values given are the average of the 3-hour values between 18:00 and 00:00 UT. The averages have then been divided into three bands representing quiet, moderate, and disturbed conditions. At frequencies of $8.01 \mathrm{MHz}$ and below, Type 1 propagation events become more frequent as $\mathrm{K}_{\mathrm{p}}$ increases, while for frequencies higher than this the percentage of nights on which this type of propagation is observed is roughly independent of $\mathrm{K}_{\mathrm{p}}$ (although there is some suggestion of a slight decrease in occurrence with increasing $\mathrm{K}_{\mathrm{p}}$ ). For Type 2 propagation, there is a clear decrease in the occurrence of observations with increasing $\mathrm{K}_{\mathrm{p}}$ for all frequencies except $4.64 \mathrm{MHz}$, where the opposite is the case. Type 4 propagation is more commonly observed at the middle range of frequencies (8.01-11.12 MHz), and becomes more frequent with increasing $\mathrm{K}_{\mathrm{p}}$. For the other propagation types, not shown in the figure, there are insufficient observations to draw any conclusions except at $4.64 \mathrm{MHz}$. For this frequency, Type 3 propagation does not appear to depend on $\mathrm{K}_{\mathrm{p}}$, while Type 5 propagation is slightly more common at higher $\mathrm{K}_{\mathrm{p}}$.

\section{Discussion and concluding remarks}

The establishment of multi-frequency HF circuits between Uppsala, Helsinki and Leicester has allowed us to observe simultaneously the direction of arrival, time of flight, Doppler shift and Doppler spread of signals reflected from the ionosphere. During the day, the measurements usually show propagation via the F-region along the GCP. At night, however, off great circle propagation often occurs which may fall into a number of categories as described above. The mechanism by which off great circle path propagation of Type 1 occurs has been investigated through simulation, the results of which are reported by in Siddle et al. (2004b). These authors concluded that propagation was likely to be via scatter from irregularities embedded within the north wall of the trough or slightly to the north in the auroral oval. The frequency of occurrence of Type 1 propagation is much lower in the recent measurements than at solar maximum and the seasonal variation has changed, for example, it was commonly observed in winter in 2001, but is not in 2006-07.

In the recent experiment the other types of propagation have either been much more common or more significant in terms of duration and azimuth deviation from the great circle than at solar maximum. At this stage of the current investigation (which is still on-going), we have not completed simulating the events so are not able to firmly propose the propagation mechanisms involved, although it anticipated that this will be the subject of a future paper. However, a few tentative suggestions are given below.

Type 2: Preliminary ray tracing has been able to reproduce the form of the azimuth deviation observed with this type of propagation. The reflection point is located in the trough (see Figure 7), but the ionospheric critical frequency is insufficient to reflect the 
signal suggesting that the signal is scattering from ionospheric irregularities in the floor of the trough. However, the modelling has not yet been able to explain why the deviations occur on one day, but not on, otherwise, very similar days.

Type 3: Since these tend to arise when the operating frequency is close to the MUF (and generally only on $4.64 \mathrm{MHz}$ ), and the azimuth deviation and TOF increase tend to be small, it is likely that these result from refraction from electron density gradients near the equatorward wall of the trough (see Figure 7).

Type 4: The simple model employed here indicates that the propagation is unlikely to be via a single ionospheric reflection. It is possible that the propagation occurs via a 2hop path with the ground reflection well to the south of the propagation path (near to the Alps, see Figure 7). A satisfactory simulation will have to explain why this type of behaviour is generally only observed at 10.39 and $11.12 \mathrm{MHz}$, just before dawn, in spring, and is more common at sunspot minimum.

Type 5: These may be due to refraction from the south wall of the trough, the coupling of elevation with azimuth (at least some of the time) suggesting that the reflection point starts out relatively close to the receiver and then moves away with time (see Figure 7). They are usually observed on the lowest frequency and more commonly in spring and winter and at sunspot minimum (the gradients in the trough walls vary with solar cycle).

It should be noted that there are some limitations to the observations, particularly when more than one propagation mode is present since the system is capable of resolving two modes only if their amplitudes differ by less than about $20 \mathrm{~dB}$. At times this is the case (e.g. see Figures 3 and 5), but at other times the presences of a strong on great circle mode may preclude the observation of a weaker off great circle mode.

It is anticipated that eventually the results from this work will enhance our understanding of HF radio propagation in the trough region and enable the propagation prediction models for this region to be improved.

\section{Acknowledgements}

The authors would like to thank the Swedish Meteorological Institute and the Finnish Meteorological Institute for hosting a transmitter at their Marsta and Nurmijärvi sites, respectively. The collaboration between the UK and Finnish groups in this investigation is facilitated through the EU COST-296 Action on the Mitigation of Ionospheric Effects on Radio Systems (MIERS). This investigation was supported by a grant from the EPSRC.

\section{References}

Halcrow, B.W. and J.S Nisbet, A model of the F2 peak electron densities in the main trough region of the ionosphere, Radio Science, 12, 815-820, 1977.

ITU-R Recommendation P1148-1, Standardized procedure for comparing predicted and observed HF sky-wave signal intensities and the presentation of such comparisons, paragraph 3.5, 1997.

Moffett, R.J. and S. Quegan, The mid-latitude trough in electron concentration of the ionospheric F-layer: a review of observations and modelling, Journal of Atmospheric and Solar Terrestrial Physics, 45, 315-343, 1983. 
Rogers N.C., E.M. Warrington and T.B. Jones, Large HF bearing errors for propagation-paths tangential to the auroral oval, IEE Proc. on Microwaves Antennas and Propagation, 14 (2), 91-96, 1997.

Siddle, D.R., A.J. Stocker and E.M. Warrington. The time-of-flight and direction of arrival of HF radio signals received over a path along the mid-latitude trough: observations. Radio Science, 39, RS4008, doi: 10.1029/2004RS003049, 2004a.

Siddle, D.R., N.Y. Zaalov, A.J. Stocker and E.M. Warrington. The time-of-flight and direction of arrival of HF radio signals received over a path along the mid-latitude trough: theoretical considerations. Radio Science, 39, RS4009, doi: 10.1029/2004RS003052, 2004b.

Stocker, A.J., E.M. Warrington and T.B. Jones, A comparison of observed and modelled deviations from the great circle direction for a $4490 \mathrm{~km}$ HF propagation path along the mid-latitude ionospheric trough, Radio Science, 38(3), 1045, doi:10.1029/2002RS002781, 2003

Warrington, E.M., T.B. Jones and B.S. Dhanda, Observations of the Doppler spread on HF signals propagated over high-latitude paths, IEE Proceedings on Microwaves, Antennas and Propagation, 144(4), 215-220, 1997.

Warrington E.M. and A.J. Stocker, Measurements of the Doppler and multipath spread of HF signals received over a path oriented along the mid-latitude trough, Radio Science, 38(5), 1080, doi:10.1029/2002RS002815, 2003.
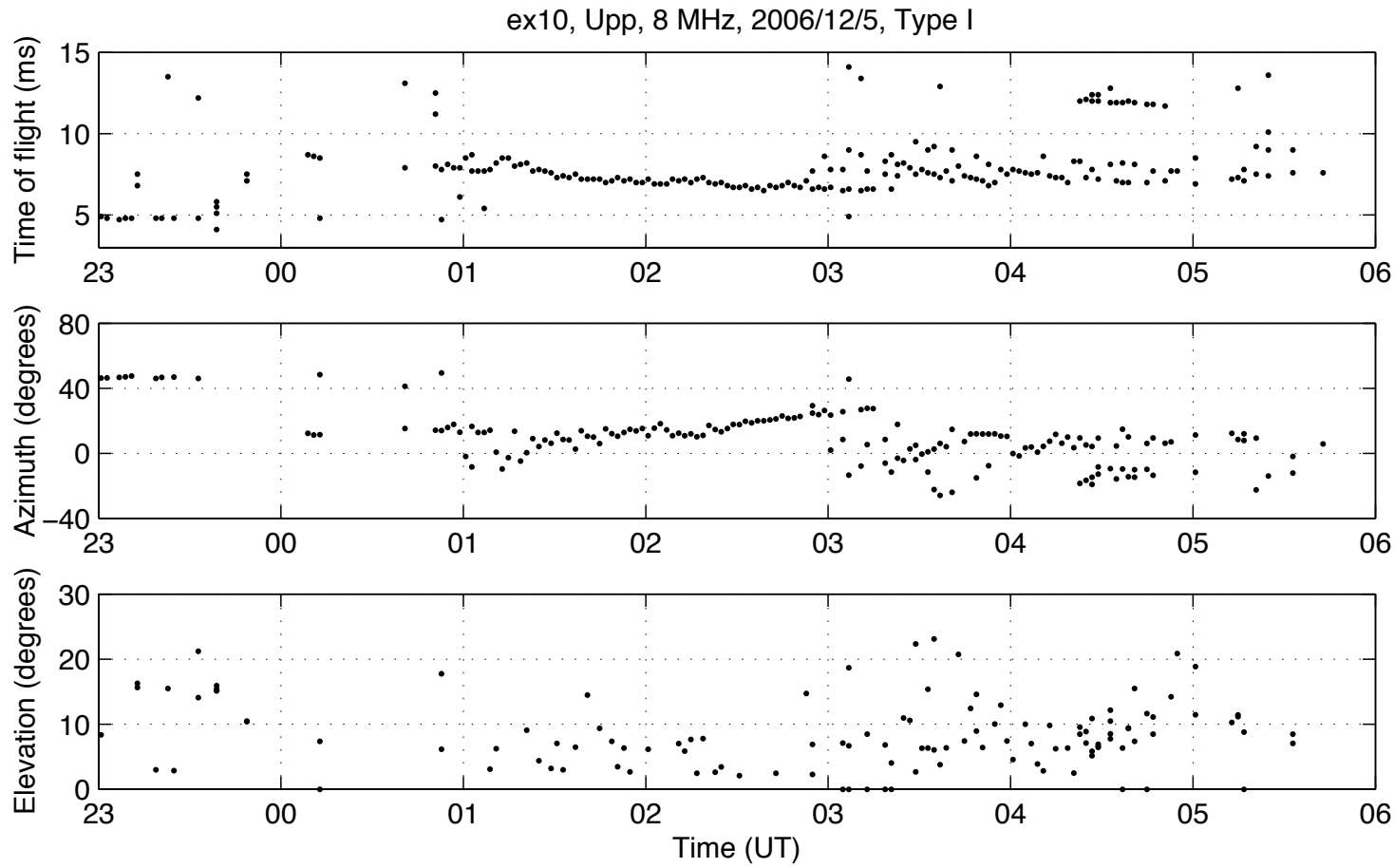

Figure 2. Observations of the (from top to bottom) time of flight, azimuth, and elevation of a signal at $8.01 \mathrm{MHz}$ on a path between Uppsala and Leicester on 5-6 December 2006 showing an example of Type 1 off great circle propagation. Note: the great circle direction is $\sim 47^{\circ}$. 

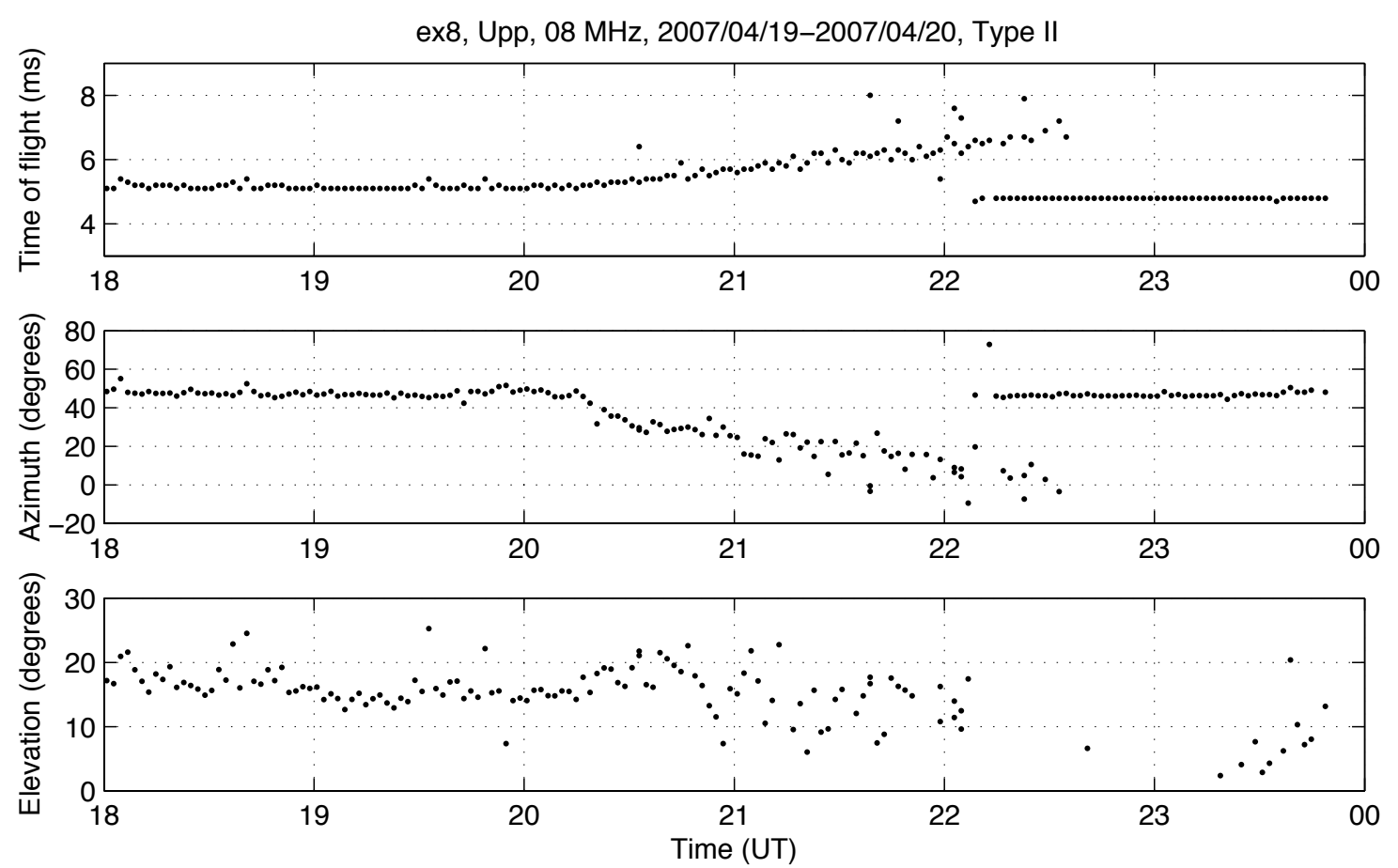

Figure 3. As Figure 2 except for 8.01 MHz signal on 19 April 2007 showing an example of Type 2 off great circle propagation. Note: the great circle direction is $\sim 47^{\circ}$.
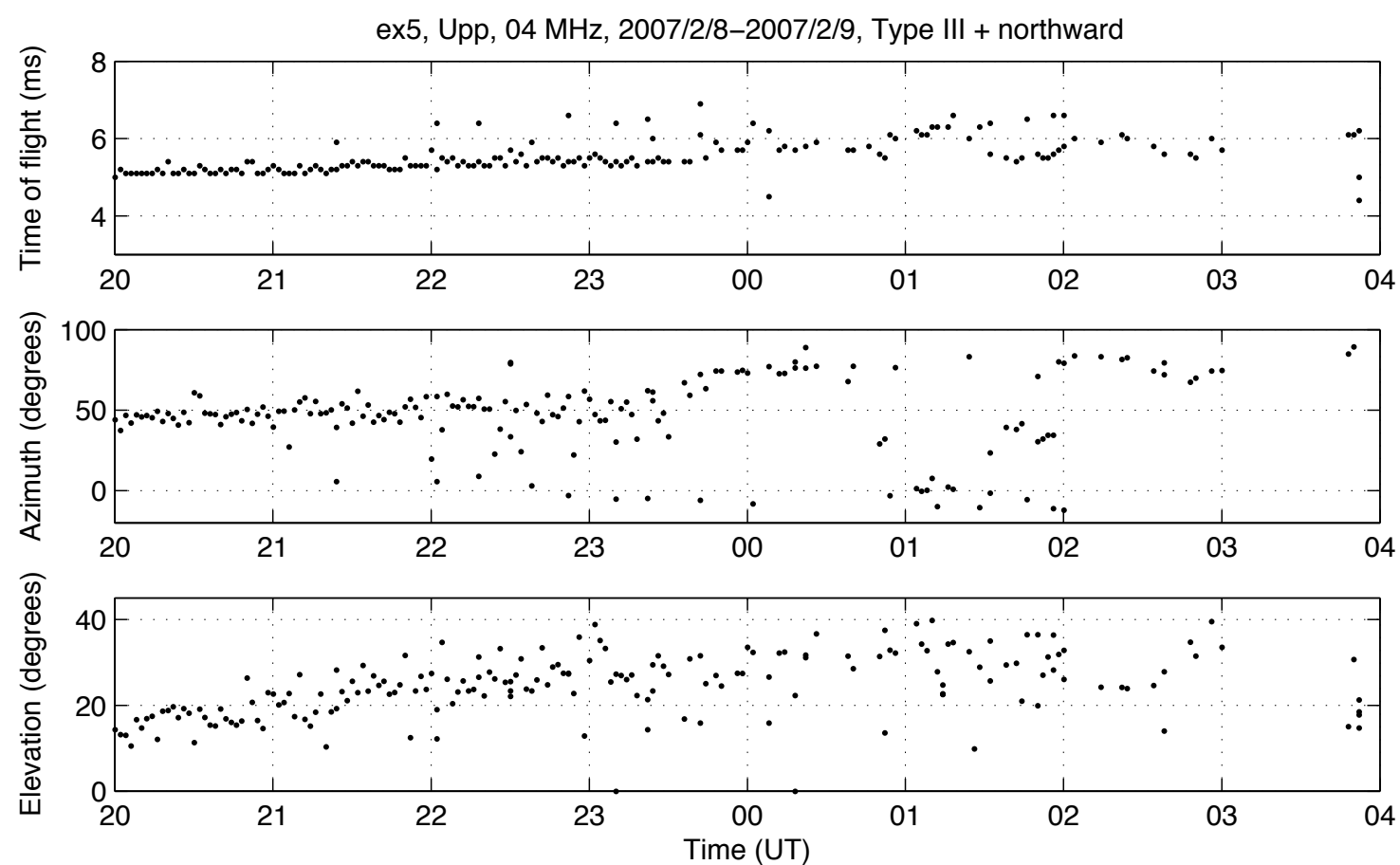

Figure 4. As for Figure 2, except for a $4.64 \mathrm{MHz}$ signal on 8-9 February 2007 and showing an example of Type 3 off great circle propagation (plus a, generally, weaker northward trace). 

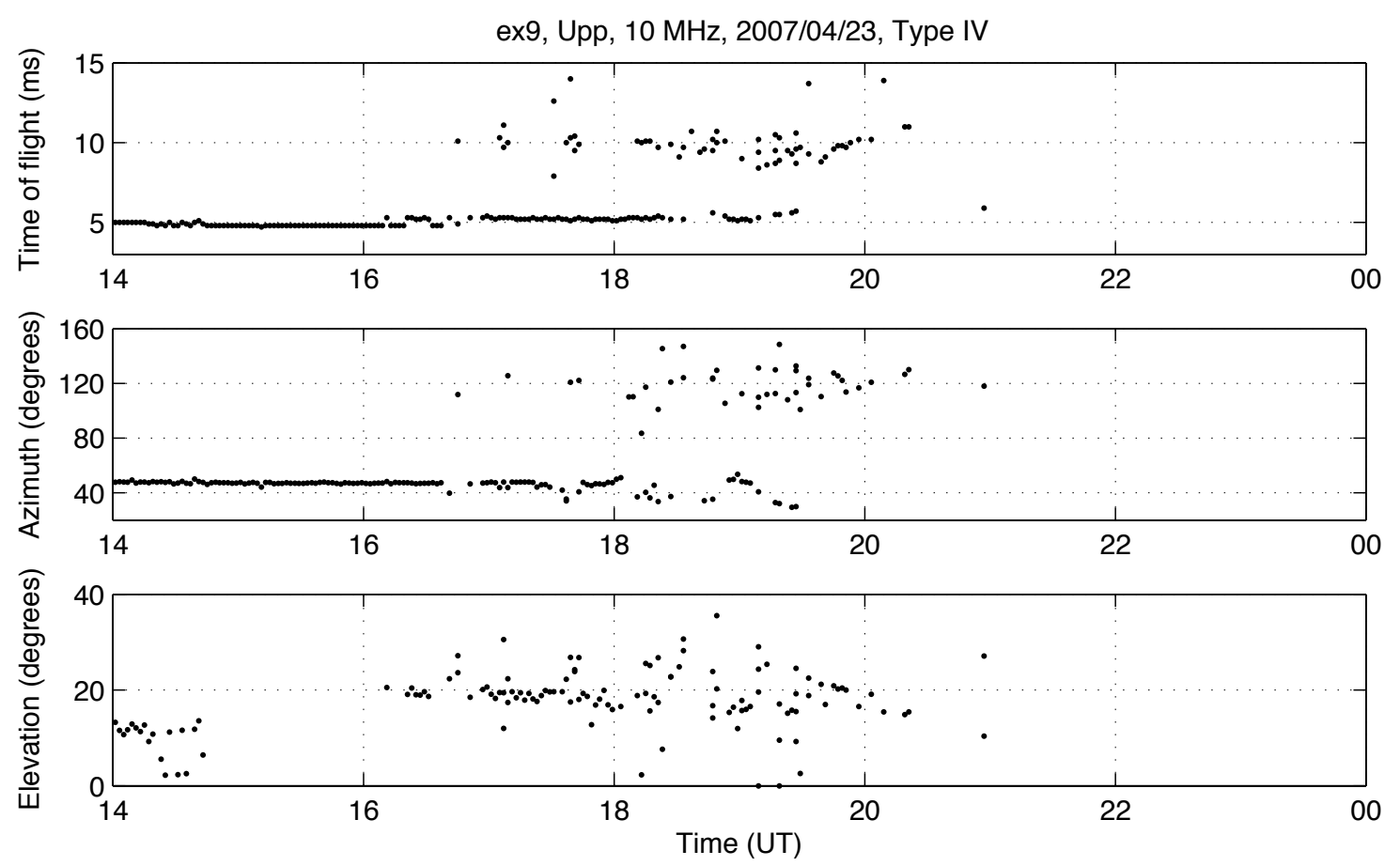

Figure 5. As for Figure 2, except for a 10.39 MHz signal on 23 April 2007 and showing an example of Type 4 off great circle propagation
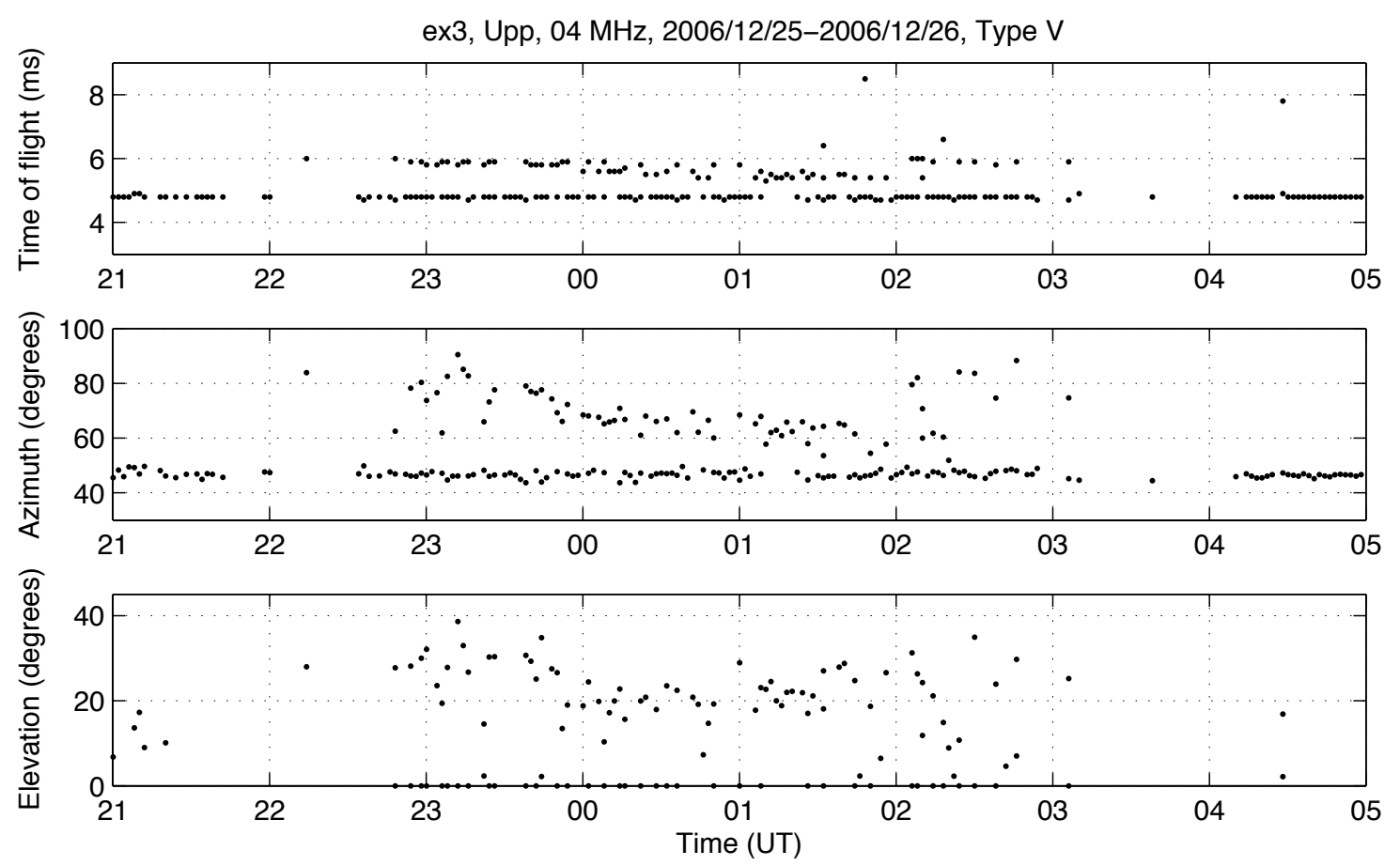

Figure 6. As for Figure 2, except for a 4.64 MHz signal on 25-26 December 2006 illustrating an example of Type 5 off great circle propagation. 

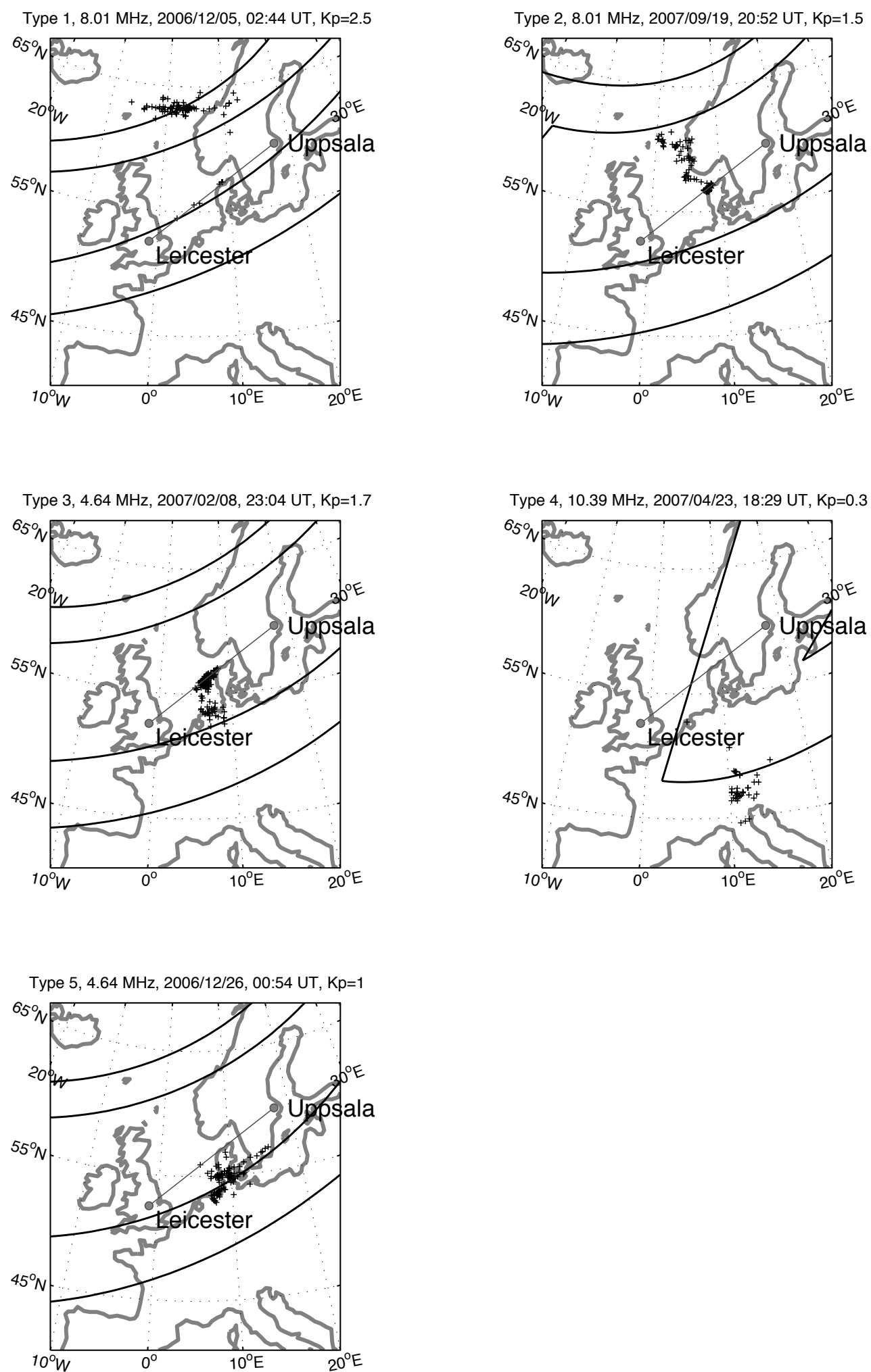

Figure 7. The reflection points derived from the observations in Figure 2 to 6 (top left, top right, middle left, middle right, and bottom left respectively). The northward signal has been omitted from the top right panel and the on great circle signal from the bottom left and middle right panels. The position of the trough during off-circle propagation has been plotted for a kp averaged from 18-00 UT (right panels) and 18-06 UT (left panels). 

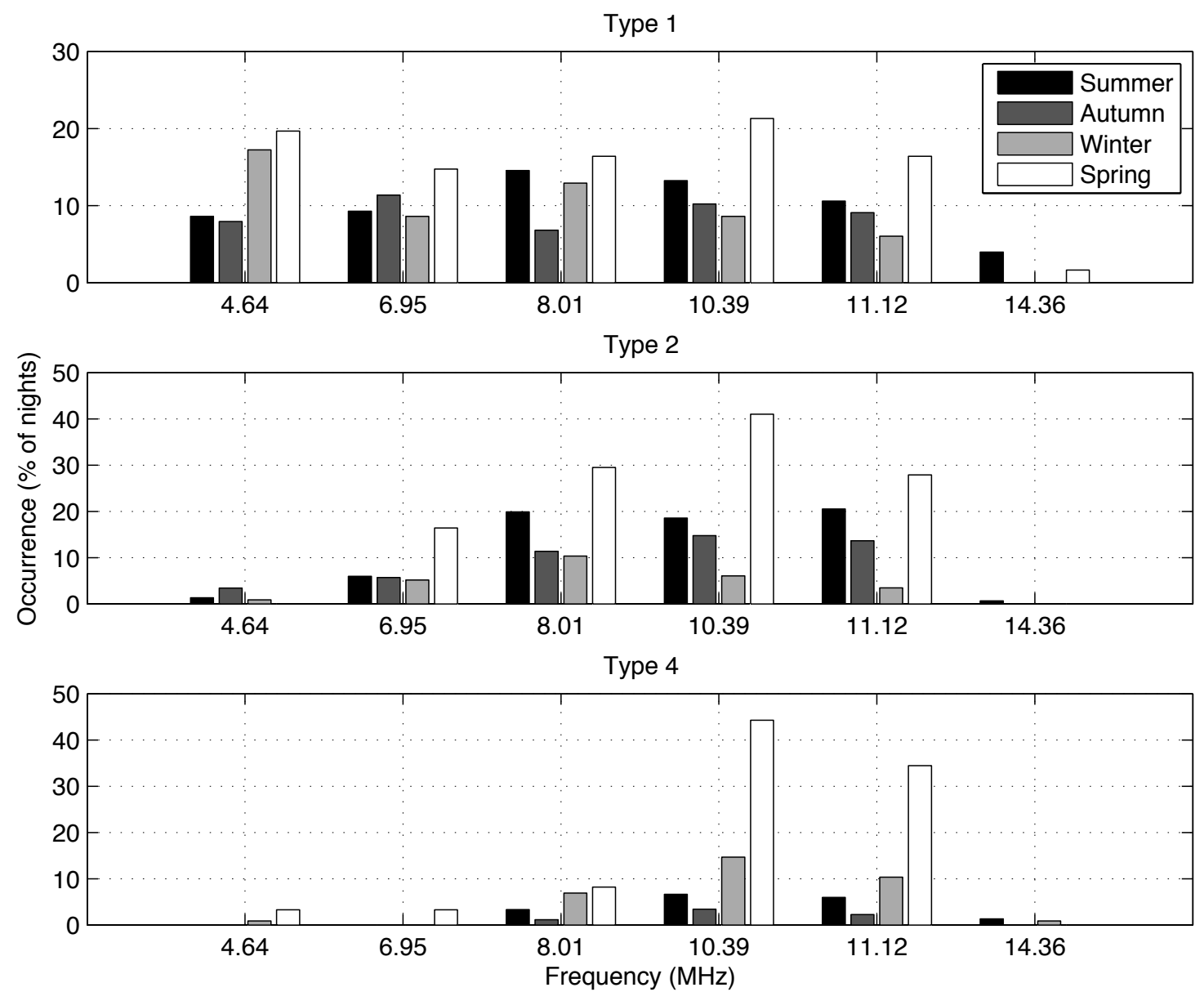

Figure 8. The percentage occurrence of (top to lower panels) Types 1, 2, and 4 off great circle propagation. Note: The number of nights observations in each season are as follows, summer $=151$, autumn $=88$, winter $=116$, and spring $=61$. 

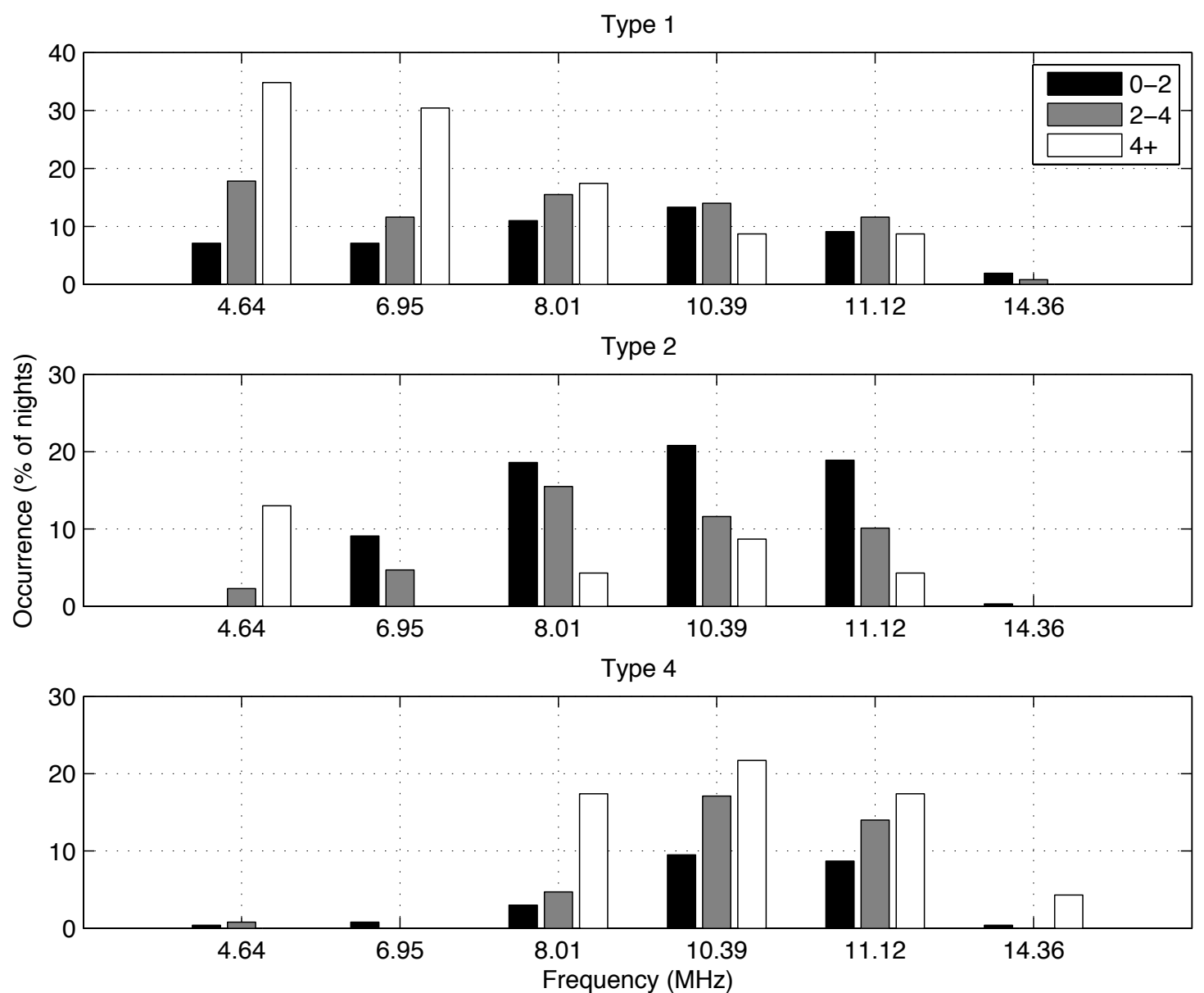

Figure 9. Occurrence of Type 1, Type 2, and Type 3 (top to lower panels, respectively) propagation as a percentage of nights for different levels of $\overline{\mathrm{kp}}$. Note: The number of nights observations at each $\overline{\mathrm{kp}}$ range are as follows $0-2=264,2-4=129$, and $4+=23$. 